\title{
Dead or alive: how the immune system detects microbial viability Matteo Ugolini ${ }^{1,2}$ and Leif E Sander ${ }^{3,4}$
}

Immune detection of microbial viability is increasingly recognized as a potent driver of innate and adaptive immune responses. Here we describe recent mechanistic insights into the process of how the immune system discriminates between viable and non-viable microbial matter. Accumulating evidence suggests a key role for microbial RNA as a widely conserved viability associated PAMP (vita-PAMP) and a molecular signal of increased infectious threat. Toll-like receptor 8 (TLR8) has recently emerged as a critical sensor for viable bacteria, ssRNA viruses, and archaea in human antigen presenting cells (APC). We discuss the role of microbial RNA, and other potential vita-PAMPs in antimicrobial immunity and vaccine responses.

\footnotetext{
Addresses

${ }^{1}$ Max Planck Unit for the Science of Pathogens, Berlin, Germany

${ }^{2}$ Max Planck Institute for Infection Biology, Berlin, Germany

${ }^{3}$ Department of Infectious Diseases and Pulmonary Medicine, CharitéUniversitätsmedizin Berlin, Corporate Member of Freie Universität Berlin, Humboldt-Universität zu Berlin, and Berlin Institute of Health, Berlin, Germany

${ }^{4}$ German Center for Lung Research (DZL), Berlin, Germany
}

Corresponding author: Sander, Leif E (leif-erik.sander@charite.de)

Current Opinion in Immunology 2019, 56:60-66

This review comes from a themed issue on Innate immunity

Edited by James Di Santo and Nicolas Manel

https://doi.org/10.1016/j.coi.2018.09.018

0952-7915/@ 2018 Elsevier Ltd. All rights reserved.

\section{Introduction}

"There is nothing over which a free man ponders less than death; his wisdom is to meditate not on death but on life"

Erwin Schrödinger, 'What is Life?'

Asked to define 'life', each branch of science is likely to present a different answer. In his collection of lectures 'What Is Life?' the Austrian physicist Erwin Schrödinger famously postulated that living organisms are distinguished from dead matter by a 'code-script', which enables them to withstand the disturbing forces of disorder (entropy) and thereby avoid decay for a period of time [1]. Pinning down the difference between living and nonliving things remains surprisingly difficult, but several defining features of 'life' are frequently mentioned. These include the ability to take up energy and transform it into growth and self-replication. Living organisms respond to their environment and generally strive to maintain the order of their internal environment, called homeostasis. Finally, all known forms of life require informational molecules, DNA and RNA. Beyond the borders of these criteria, there is a grey zone of creatures that lack some defining features of 'life', yet they might still be considered 'viable'. Viruses, for instance, rely on other organisms for replication and metabolism and have been described as living 'a kind of borrowed life' [2]. Numerous pathogens have adopted an obligate intracellular 'lifestyle' characterized by an inability to grow outside of their host cells; and dormant life forms, such as bacterial endospores, are non-reproductive, metabolically inactive, yet they retain the capacity to revitalize and reacquire all characteristics of living cells, and most importantly, they maintain the ability to infect their hosts. Immune defense systems evolved in response to these threats from all domains of microbial life and its grey zones. For the sake of maintaining homeostasis, it appears that the magnitude and the quality of antimicrobial immune responses are precisely proportionate to the infectious threat. Efficient host defense therefore requires meticulous risk assessment of microbial contacts [3]. Based on this conceptual framework, we have previously proposed that the immune system perceives 'microbial viability' per se as an indicator of increased infectious threat $[3,4]$. Here, we discuss the immune system's fundamental capacity to discriminate viable from non-viable microbial material. We review the most recent insights into the molecular mechanisms of 'viability-sensing' in different species and its effects on antimicrobial immunity.

\section{Immune recognition beyond 'self' versus 'non- self'}

Pattern recognition arguably forms the basis of 'self' versus 'non-self' discrimination by the innate immune system [5]. Pattern recognition receptors (PRRs) detect conserved molecular patterns (PAMPs) of microorganisms, and PRR activation controls important functions of antigen presenting cells (APC), with profound effects on subsequent adaptive immune responses [6]. However, further distinctions beyond the binary categories of 'self' 
and 'non-self' are necessary for accurate gaging of the infectious threat, in order to elicit an adequately measured immune response. It has become increasingly clear that the specific context of PRR engagement and the nature of their ligands critically shapes the response to a given microbial encounter. Based on observations that most pathogens tend to employ common strategies to cause infection and disease [7], Vance et al. proposed that the immune system distinguishes disease-causing pathogens from less virulent microbes by recognizing such conserved pathogenic activities as 'patterns of pathogenesis' [4]. Besides cytosolic invasion and active perturbation of cellular organization, the authors identified 'growth' as a common pathogenic pattern. They postulated the existence of specific PAMPs that would convey either bacterial life or death, respectively, to the immune system. Interestingly, they speculated that the release of macromolecules such as DNA and RNA would be indicative of bacterial death [4].

Supporting the idea of designated PAMPs that signify bacterial cell death, it was recently reported that polymeric peptidoglycan (PGN) released from the cell walls of dying bacteria binds to a regulatory isoform of the PGN-recognition protein-LC (rPGRP-LC) and inhibits the immune deficiency (IMD) pathway in Drosophila. In contrast, monomeric peptidoglycan (PGN), a byproduct of bacterial growth, activates the IMD pathway leading to antimicrobial peptide production and inflammation. Bacterial lysis, mediated by the secreted antimicrobial peptides, causes the release of polymeric PGN, thus providing an inhibitory feedback via rPGRP-LC. The study implies that immune recognition of dead bacteria contributes to the resolution of inflammation in the aftermath of bacterial infections in flies [8]

\section{Sensing bacterial viability}

Epidemiological and empirical data have long suggested that living microorganisms, such as the ones included in live attenuated vaccines, induce more robust immune responses than their killed counterparts in vertebrates [9$11,12^{\circ}$ ]. Pamer and colleagues noted that vaccination with heat-killed Listeria moncytogenes failed to prime effector $\mathrm{CD}^{+} \mathrm{T}$ cells in mice $\left[12^{\circ \bullet}\right]$, and several other studies have also reported a superior ability of viable bacteria to elicit protective immunity [13-15]. Expanding upon some of these earlier observations, we have recently uncovered an inherent ability of innate immune cells to discriminate living from dead bacteria, independently of their virulence. In murine macrophages and dendritic cells (DC), detection of live bacteria led to activation of the NLRP3 inflammasome complex and the subsequent release of IL-1 $\beta$, and inflammatory cell death (pyroptosis), as well as increased production of type-I Interferon (IFN) $\left[16^{\circ}\right]$. These unique responses to live bacteria were dependent on the TLR signaling adaptor molecule TIR-domain-containing adapter inducing IFN- $\beta$
(TRIF), which suggested a role for pattern recognition in the detection of living bacteria. While replication deficient nutritional auxotrophic bacteria (Escherichia coli), and even paraformaldehyde-inactivated bacteria induce aforementioned TRIF-mediated responses, bacterial killing by heat, antibiotic treatment, or UV irradiation abrogates inflammasome activation [16 $\left.{ }^{\bullet \bullet}\right]$. Importantly, when mixed with living bacteria, heat killed bacteria do not affect the enhanced responses to living bacteria. Collectively, these data suggest the existence of fixable molecules (PAMPs) that associate specifically with viable bacteria and that elicit distinct immune responses. Bacterial RNA was identified as such a 'viability-associated PAMP' (vita-PAMP), the detection of which triggers a state of alert not warranted for dead bacteria $\left[3,16^{\circ}\right]$.

\section{Microbial RNA - a versatile signal of microbial threat}

RNA sensors have been extensively studied in the context of anti-viral defense, with particular focus on type-I IFN responses initiated by cytosolic helicases retinoic acid-inducible gene I (RIG-I) and Melanoma Differentiation-Associated protein 5 (MDA5), or sensors with direct antiviral activity including protein kinase $\mathrm{R}$ (PKR), 2'-5'-oligoadenylate synthetase 1 (OAS1), and ribonuclease L (RNase L) [17]. Several recent publications have highlighted the hitherto largely overlooked role of RNA receptors in antibacterial immunity [18]. Bacterial RNA is rapidly released and degraded in dying bacteria, whereas bacterial DNA is remarkably stable after bacterial cell death, and can be found in the remains of tissues even centuries after bacterial infection [19]. When added together with killed bacteria, bacterial RNA restores NLRP3 inflammasome activation and IFN- $\beta$ production in mouse macrophages and DC to the same levels elicited by viable bacteria $\left[16^{\bullet \bullet}\right]$. Human monocytes and myeloid DC have a similar ability to discriminate viable from killed bacteria. Detection of bacterial RNA activates a transcriptional signature, with selective expression of $T N F$ and $I / 12 B$ in response to living bacteria $\left[20^{\circ \bullet}\right]$ and microbial RNA [21]. Selective induction of IL-12 is conserved in porcine monocytes and DC, whereas murine APC produce IL-12 and TNF in response to a broad variety of inanimate microbial PAMPs $\left[16^{\bullet \bullet}, 20\right]$. Conversely, IL- $1 \beta$ production is specifically induced upon detection of viable bacteria and bacterial RNA in both human and murine APC $\left[16^{\circ \bullet}\right.$ $\left., 20^{\bullet \bullet}, 22\right]$. Interestingly, bacterial spores, although metabolically inactive, also contain high amounts of RNA and activate inflammatory host responses in humans and mice, indicating that they are accurately perceived as infectious threats by the immune system [23]. Detection of bacterial RNA as a vita-PAMP thus appears to represent a conserved stimulus for escalated immune responses, albeit the responses are different in humans and mice. Sensing of live bacteria and bacterial RNA in human and porcine APC requires TLR8, a highly 
conserved endosomal receptor for single-stranded RNA (ssRNA) $\left[20^{\bullet},\right]$ (Figure 1). Despite being expressed in various cell types, murine TLR8 is irresponsive to ssRNA [25]. Instead, TLR13 has been shown to sense bacterial $23 \mathrm{~S}$ ribosomal RNA in mice $[26,27]$. Its role in the recognition of bacterial viability in mice is unclear, and TLR13 is missing in the human genome [28] (Figure 1). Its endosomal localization in APC ideally positions TLR8 to surveil phagocytic cargo for microbial RNA, and hence signals of microbial viability. Still several questions regarding the molecular mechanisms of TLR8 activation by bacterial RNA remain unanswered. The recent resolution of the molecular structure of TLR 8 in complex with its natural ligand has revealed two distinct ligand interaction sites that actually bind degradation products of ssRNA, uridine (U) nucleosides and a U-containing small oligonucleotide, respectively $\left[29^{\circ}\right]$. These new insights pose the question as to how natural TLR8 ligands are generated from microbial RNA. This process is likely to involvespecific endolysosomal RNases and phosphatases
(Figure 1), and it will be important to characterize these enzymes and their potential regulation by other co-factors. Immune detection of highly conserved molecules like RNA harbors an inherent hazard of causing self-reactivity [30]. Activation of TLR8 by self-RNA is prevented on multiple levels. Ligand accessibility is restricted by segregating TLR8 and endogenous RNA into different subcellular compartments, that is exclusion of RNA from endosomes. Availabity of RNA, for example during efferocytosis, is minimized by rapid degradation of endogenous RNA [31,30]. In addition, TLR8 activation by residual self-RNA is prevented by inhibitory nucleoside modifications. Mammalian rRNA, for instance, contains around ten times more pseudoruridines and 25 times more 2'-O-methylated nucleosides than bacterial rRNA, which makes mammalian rRNA generally a very poor TLR8 activator [32]. Mammalian mRNA contains numerous additional modifications like 5-methylcytidine and N6methyladenine, both of which also strongly inhibit TLR8 activation [32]. Bacterial mRNA, on the

\section{Figure 1}

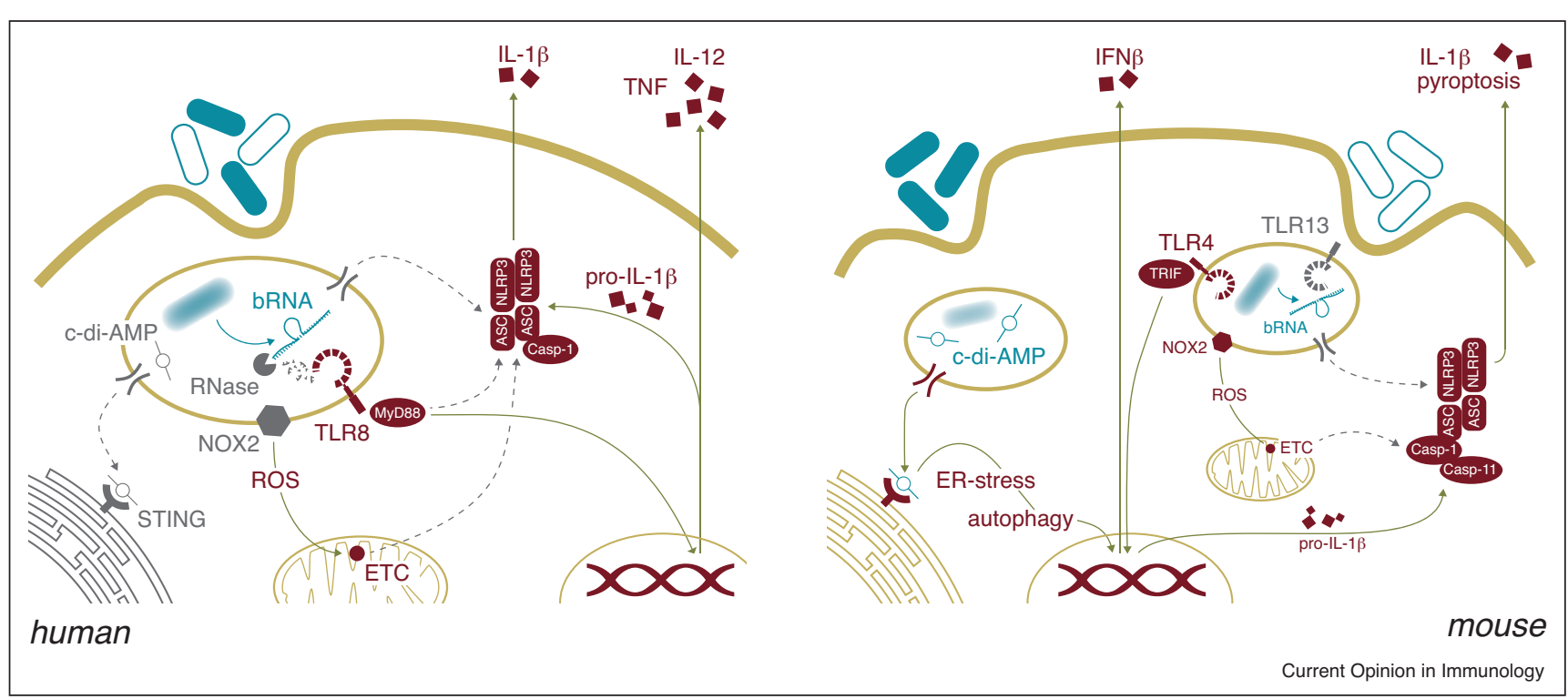

Recognition of bacterial viability in human and murine APC. Left: Human APC engulf viable bacteria (filled symbols: Gram-positive, open symbols: Gram-negative). Degradation in the phagolysosome is thought to liberate bacterial RNA (bRNA) and other bacterial ligands. bRNA is further degraded, possibly by so far uncharacterized endosomal RNAses and phosphatases. Degradation products of bRNA are sensed by TLR8, which induces transcription of IL-12 and TNF, as well as pro-IL1 $\beta$ and other components of the NLRP3 inflammasome. Subsequent activation of the NLRP3 inflammasome leads to cleavage and release of IL-1 $\beta$. The mechanisms of RNA-mediated NLRP3 activation are not clear, and could involve direct cytosolic sensing of bRNA exiting from the endosome either by leakage or active translocation, or indirect alternative activation via TLR8-dependent signals. RNA mediated generation of phagosomal ROS and subsequent adaptations of the mitochondrial ETC contribute to NLRP3 inflammasome activation via an unknown mechanism. The role of STING mediated sensing of bacterial cyclic-di-nucleotides in responses to viable bacteria has not been investigated in human APC. Right: Mouse APC detect viability of Gram-positive and Gram-negative bacteria through distinct pathways. Gram-negative bacteria induce TLR4/TRIF-dependent and bRNA-dependent IFN responses and activation of the NLRP3 inflammasome. Inflammasome assembly requires TRIF-IFN $\beta$-dependent licensing of Caspase-11, leading to non-canonical inflammasome activation, cleavage and release of IL-1 $\beta$ and pyroptosis. Phagocytosis of Gram-negative bacteria also promotes NOX2-mediated phagosomal ROS production, which is involved in metabolic reprogramming and mitochondrial ETC adaptations. Enhanced ETC CII activity contributes to NLRP3 inflammasome activation through an hitherto unknown pathway, potentially involving mitochondrial ROS. TLR13 senses bacterial rRNA, but its role in 'viability sensing' has not been investigated. Viable Gram-positive bacteria release c-di-AMP, which activates STING and triggers a coordinated host response involving ER stress and subsequent auto-phagocytic removal of ER membranes and production of IFN $\beta$. Pathways whose role is not fully characterized are depicted in gray symbols and gray dotted lines. 
other hand, is virtually devoid of known nucleoside modifications, with the exception of a $5^{\prime}$-nicotinamide adenine dinucleotide (NAD)-cap structure, found in a subset of bacterial mRNA transcripts [33]. In addition, TLR8 displays a certain level of sequence preference for GU-rich ssRNA [34], whereas 3'-polyadenylation appears to inhibit TLR activation by ssRNA [21]. Inflammasome activation by bacterial mRNA in mice is also inhibited by $3^{\prime}$ polyadenylation $\left[16^{\circ}\right]$. Intriguingly, both human and murine APC respond to prokaryotic mRNA, leading to NLRP3 inflammasome activation, whereas bacterial rRNA and small RNAs only activate the inflammasome in human, but not in murine APC $\left[16^{\bullet \bullet}, 22\right]$. The discrepant reactogenity of some bacterial RNA species in humans and mice suggests slightly distinct sensing or transport pathways upstream of NLRP3 inflammasome assembly (Figure 1). So far, the exact mechanisms of NLRP3 activation in response to bacterial RNA remain incompletely resolved. In murine APC, it appears that several pathways collude, involving TRIF-dependent production of IFN- $\beta$, auto-/paracrine induction of Caspase-11 and non-canonical inflammasome activation (Reference PMC3660860). It was shown that fractions of phagolysosomal contents translocate to the cytosol during bacterial phagocytosis, yet it is unclear whether this communication and potential transfer of bacterial RNA is required for inflammasome activation (Figure 1)(REF 16). A recent study revealed an unexpected TLR8 dependent mode of inflammasome activation in human monocytes stimulated with the archeon Methanosphaera stadtmanae, a constituent of the human microbiome [ $\left.35^{\circ}\right]$. In fact, all inflammatory responses to M. stadtmanae were shown to rely on TLR8 and recognition of archaeal RNA, suggesting that microbial viability might be critical for immune recognition of archaea, yet this remains to be investigated. The role of TLR8 for NLRP3 inflammasome activation and IL- $1 \beta$ release in response to bacteria is currently under investigation (Figure 1). Often disregarded because of its dysfunction in mice, TLR8 has lately emerged as one of the most versatile innate immune sensors of microbial RNA, which is recognized as a conserved molecular signature of viable bacteria, archaea, and ssRNA viruses $\left[18,20^{\bullet \bullet}, 34,35^{\bullet}\right]$.

\section{Metabolic responses to bacterial RNA}

Microbial infections cause profound metabolic reprogramming of innate immune cells, in order to promote inflammation and pathogen clearance, and eventually restoration of tissue homeostasis [36,37]. Macrophages exposed to LPS or killed bacteria undergo a glycolytic switch, characterized by decreased mitochondrial respiration and enhanced glycolysis. Interestingly, recognition of live bacteria and bacterial RNA induces rapid modulations of the mitochondrial electron transport chain (ETC). Whereas both live and killed bacteria induce a lower ETC complex I (CI) activity, only live bacteria evoke a concomitantly increased activity of CII and as a result, the accumulation of fumarate, an intermediate in the tricarboxylic acid cycle with antimicrobial and immunomodulatory activities $\left[38^{\bullet \bullet}\right]$. The observed mitochondrial ETC adaptations required TLR-induced reactive oxygen species (ROS), generated by the phagosomal NAPDH oxidase 2 (NOX2). Increased electron flow from CII promotes mitochondrial ROS formation and inflammatory immune responses, including NLRP3 inflammasome activation [39] (Figure 1). Blocking CII activity during phagocytosis of live bacteria reverses inflammatory cytokine production [ $\left.38^{\bullet \bullet}\right]$. These findings establish a link between the recognition of bacterial RNA, mitochondrial respiration, and inflammatory cytokine production.

\section{Other vita-PAMPs}

Other molecular signatures of microbial viability (=vitaPAMPs) besides RNA, are likely to exist. Possible candidates include byproducts of bacterial metabolites and signaling molecules like second-messengers, signal peptides [40] and quorum sensing molecules [41]. It was recently reported that cyclic-di-adenosine monophosphate (c-di-AMP), a second messenger molecule in Gram-positive bacteria, is recognized as a vita-PAMP in murine macrophages [42]. It has been noted earlier that c-di-AMP directly binds and activates Stimulator of IFN genes (STING) [43], a sensor of endogenous cyclic di-nucleotide cGMP-cAMP (cGAMP) produced in response to cytosolic DNA [44]. Located on the endoplasmic reticulum, STING activation induces ER membrane stress. This triggers mTOR inactivation and enhanced autophagy of damaged ER membranes, sometimes referred to as ER-phagy [45]. Removal of damaged ER prevents cell death and additionally orchestrates an IFN response by redistributing ER-resident STING to autophagosomal membranes $[42,46]$ (Figure 1). The contribution of listeriolysin- $O$ (LLO, encoded by $H l y$ )-mediated cytosolic invasion and release of c-di-AMP for STING activation is not fully understood. $H / y$-deficient Listeria monocytogenes mutants, although fully viable, failed to activate STING and IFN responses upon injection into mice [47]. In contrast, non-invasive L.innocua were shown to activate STING, autophagy, and IFN responses in macrophages and DC, as long as the bacteria were viable [42]. Thus, it remains to be further investigated whether bacterial cyclic di-nucleotides generally function as vita-PAMPs, especially in human phagocytes (Figure 1).

\section{Impact of vita-PAMPs on immunity}

Detection of microbial viability has effects beyond classical APC like monocytes, macrophages and DC. Recognition of live bacteria or bacterial RNA induces enhanced NETosis [48], a highly inflammatory form of cell death in neutrophils that is characterized by the expulsion of nuclear DNA and proteins, so called neutrophil-extracellular traps (NETs) [49]. Limiting highly inflammatory and damaging responses like NETosis to encounters of acute infectious threat may be required for tissue 
homeostasis, although many microbial triggers of NETosis have been described [49].

Besides cells of the innate immune system, there are $\mathrm{T}$ cells that specialize in detecting viable microorganisms. Mucosal associated invariant T cells (MAIT cells), a large population of semi-invariant $\mathrm{T}$ cells, respond to live but not to killed bacteria $\left[50^{\circ}\right]$. MAIT cells recognize an intermediate in the microbial biosynthesis of riboflavin (vitamin B2) as their cognate antigen, which is not found in dead microbes or in host cells $\left[51^{\bullet \bullet}\right]$.

Conventional $\mathrm{T}$ cell and $\mathrm{B}$ cell responses are also regulated following innate immune recognition of microbial viability. Activation of TLR8 in human APC by live bacteria, bacterial RNA, or nucleoside analogues is a potent driver of $\mathrm{T}$ follicular helper $\left(\mathrm{T}_{\mathrm{FH}}\right)$ cell differentiation and vaccine responses $\left[20^{\circ \bullet}\right]$. $\mathrm{T}_{\mathrm{FH}}$ cells represent a specialized $\mathrm{T}$ helper cell subset with a critical role in germinal center reactions, B cell memory, and humoral immunity. Vaccination with live bacteria enhances the generation of $\mathrm{T}_{\mathrm{FH}}$ cells and humoral immune responses in swine and in mice $\left[20^{\bullet \bullet}, 24\right]$. Moreover, a hypermorphic TLR8 single-nucleotide polymorphism is associated with improved protective immunity elicited by vaccination with a live bacterial vaccine, bacillus Calmette-Guérin $(\mathrm{BCG})$, in a human cohort $\left[20^{\circ}\right]$. These new findings may help to explain the often-observed superiority of live attenuated vaccines at inducing long-lived protective immunity.

Finally, TLR8 seems to play a unique role in neonatal immunity. Neonatal phagocytes display a profound hyporesponsiveness to most microbial stimuli - except for TLR8 ligands [52]. Agonists of TLR8 can significantly boost otherwise notoriously blunted neonatal vaccine responses [53]. Restricting inflammatory immune responses to a minimum might be advantageous in early life, in order to permit colonization of body surfaces by beneficial microflora, while reducing the risk of invasive infection via surveillance of the cell interior for traces of microbial life.

\section{Concluding remarks}

Immune detection of microbial viability is increasingly recognized as a potent driver of innate and adaptive immune responses. Here we describe recent mechanistic insights into this process. Accumulating evidence suggests a key role for microbial RNA as a widely conserved vita-PAMP and a molecular signal of increased infectious threat. TLR8 has been identified as an important sensor for viable bacteria, archaea, and certain viruses, but further clarification of the cellular recognition pathways and their impact on antimicrobial immunity is needed. Most of the studies presented in this review dissected antibacterial responses, and it will be important to expand the current findings to other relevant microbes, especially those included in common 'live attenuated' vaccines. Despite recent advances, the concept of 'viabilityrecognition' will clearly require further experimental exploration. Some of our current views may need to be revised in the future, which takes us back to Schrödinger and 'What is Life?': "If a man never contradicts himself, the reason must be that he virtually never says anything at all." (Erwin Schrödinger)

\section{Conflict of interest statement}

Nothing declared.

\section{Acknowledgement}

L.E.S. is supported by the German Research Council (DFG grant SA19402/1, SFB-TR84TP C08 and C10)

\section{References and recommended reading}

Papers of particular interest, published within the period of review, have been highlighted as:

- of special interest

$\bullet$ of outstanding interest

1. Schrödinger E: What is Life? The Physical Aspect of the Living Cell. Cambridge University Press; 1944.

2. van Regenmortel $\mathrm{MH}$, Mahy BW: Emerging issues in virus taxonomy. Emerg Infect Dis 2004, 10:8-13.

3. Blander JM, Sander LE: Beyond pattern recognition: five immune checkpoints for scaling the microbial threat. Nat Rev Immun 2012, 12:215-225.

4. Vance RE, Isberg RR, Portnoy DA: Patterns of pathogenesis: discrimination of pathogenic and nonpathogenic microbes by the innate immune system. Cell Host Microbe 2009, 6:10-21.

5. Medzhitov R: Approaching the asymptote: $\mathbf{2 0}$ years later. Immunity 2009, 30:766-775.

6. Iwasaki A, Medzhitov R: Regulation of adaptive immunity by the innate immune system. Science 2010, 327:291-295.

7. Finlay BB, Falkow S: Common themes in microbial pathogenicity revisited. Microbiol Mol Biol Rev 1997, 61:136169.

8. Neyen C, Runchel C, Schupfer F, Meier P, Lemaitre B: The regulatory isoform rPGRP-LC induces immune resolution via endosomal degradation of receptors. Nat Immunol 2016, 17:1150-1158.

9. Cheers $\mathrm{C}$, Zhan $\mathrm{Y}$ : How do macrophages distinguish the living from the dead? Trends Microbiol 1996, 4:453-455.

10. Kawamura I, Yang J, Takaesu Y, Fujita M, Nomoto K, Mitsuyama M: Antigen provoking gamma interferon production in response to Mycobacterium bovis BCG and functional difference in $\mathrm{T}$-cell responses to this antigen between viable and killed BCG-immunized mice. Infect Immun 1994, 62:43964403.

11. Rauh LW, Schmidt R: Measles immunization with killed virus vaccine. Serum antibody titers and experience with exposure to measles epidemic. Am J Dis Child 1965, 109:232-237.

12. Lauvau G, Vijh S, Kong P, Horng T, Kerksiek K, Serbina N,

-• Tuma RA, Pamer EG: Priming of memory but not effector CD8 T cells by a killed bacterial vaccine. Science 2001, 294:17351739.

Landmark study demonstrating a failure of heat killed Listeria monocytogenes at priming effector CD8 T cell responses.

13. Brockstedt DG, Bahjat KS, Giedlin MA, Liu W, Leong M, Luckett W, Gao Y, Schnupf P, Kapadia D, Castro G et al.: Killed but metabolically active microbes: a new vaccine paradigm for eliciting effector T-cell responses and protective immunity. Nat Med 2005, 11:853-860. 
14. Coward C, Restif O, Dybowski R, Grant AJ, Maskell DJ, Mastroeni $P$ : The effects of vaccination and immunity on bacterial infection dynamics in vivo. PLoS Pathog 2014, 10: e1004359.

15. Liu X, Yang MJ, Wang SN, Xu D, Li H, Peng XX: Differential antibody responses to outer membrane proteins contribute to differential immune protections between live and inactivated vibrio parahemolyticus. J Proteome Res 2018, 17:2987-2994.

16. Sander LE, Davis MJ, Boekschoten MV, Amsen D, Dascher CC,

-• Ryffel B, Swanson JA, Muller M, Blander JM: Detection of prokaryotic mRNA signifies microbial viability and promotes immunity. Nature 2011, 474:385-389.

Formal demonstration of the capacity of innate immune cells to distinguish between viable and killed bacteria through the recognition of prokaryotic RNA.

17. Chow KT, Gale M Jr, Loo Y-M: RIG-I and other RNA sensors in antiviral immunity. Annu Rev Immun 2018, 36:667-694.

18. Eigenbrod T, Dalpke AH: Bacterial RNA: an underestimated stimulus for innate immune responses. J Immun 2015, 195:411418.

19. Bos KI, Schuenemann VJ, Golding GB, Burbano HA, Waglechner N, Coombes BK, McPhee JB, DeWitte SN, Meyer M, Schmedes $S$ et al.: A draft genome of Yersinia pestis from victims of the Black Death. Nature 2011, 478:506.

20. Ugolini M, Gerhard J, Burkert S, Jensen KJ, Georg P, Ebner F

-• Volkers SM, Thada S, Dietert K, Bauer L et al.: Recognition of microbial viability via TLR8 drives TFH cell differentiation and vaccine responses. Nat Immunol 2018, 19:386-396.

Identification of TLR8 as a vita-PAMP receptor in human APC, stimulation of which drives $\mathrm{T}$ follicular helper cell and vaccine responses.

21. Koski GK, Karikó K, Xu S, Weissman D, Cohen PA, Czerniecki BJ: Cutting edge: innate immune system discriminates between RNA containing bacterial versus eukaryotic structural features that prime for high-level IL-12 secretion by dendritic cells. J Immun 2004, 172:3989-3993.

22. Sha W, Mitoma H, Hanabuchi S, Bao M, Weng L, Sugimoto N Liu Y, Zhang Z, Zhong J, Sun B et al.: Human NLRP3 inflammasome senses multiple types of bacterial RNAs. Proc Nat Acad Sci 2014, 111:16059-16064.

23. Choo MK, Sano Y, Kim C, Yasuda K, Li XD, Lin X, StenzelPoore M, Alexopoulou L, Ghosh S, Latz E et al.: TLR sensing of bacterial spore-associated RNA triggers host immune responses with detrimental effects. J Exp Med 2017 214:1297-1311.

24. Barbet G, Sander LE, Geswell M, Leonardi I, Cerutti A, Iliev I, Blander JM: Sensing microbial viability through bacterial RNA augments $\mathrm{T}$ follicular helper cell and antibody responses. Immunity 2018, 48:584-598 e585.

25. Gorden KK, Qiu XX, Binsfeld CC, Vasilakos JP, Alkan SS: Cutting edge: activation of murine TLR8 by a combination of imidazoquinoline immune response modifiers and polyT oligodeoxynucleotides. J Immunol 2006, 177:6584-6587.

26. Oldenburg M, Kruger A, Ferstl R, Kaufmann A, Nees G, Sigmund A, Bathke B, Lauterbach H, Suter M, Dreher S et al.: TLR13 recognizes bacterial 23S rRNA devoid of erythromycin resistance-forming modification. Science 2012, 337:11111115.

27. Hidmark A, von Saint Paul A, Dalpke AH: Cutting edge: TLR13 is a receptor for bacterial RNA. J Immunol 2012, 189:2717-2721.

28. Signorino G, Mohammadi N, Patane F, Buscetta M, Venza M, Venza I, Mancuso G, Midiri A, Alexopoulou L, Teti G et al.: Role of Toll-like receptor 13 in innate immune recognition of group $B$ streptococci. Infect Immun 2014, 82:5013-5022.

29. Tanji H, Ohto U, Shibata T, Taoka M, Yamauchi Y, Isobe T,

- Miyake K, Shimizu T: Toll-like receptor 8 senses degradation products of single-stranded RNA. Nat Struct Mol Biol 2015 22:109-115.

Resolution of the crystal structure of TLR8 demonstrating an unexpected mode of activation by degradation of products of RNA.
30. Roers A, Hiller B, Hornung V: Recognition of endogenous nucleic acids by the innate immune system. Immunity 2016 , 44:739-754.

31. Schlee M, Hartmann G: Discriminating self from non-self in nucleic acid sensing. Nat Rev Immunol 2016, 16:566-580.

32. Kariko K, Buckstein M, Ni H, Weissman D: Suppression of RNA recognition by Toll-like receptors: the impact of nucleoside modification and the evolutionary origin of RNA. Immunity 2005, 23:165-175.

33. Cahová H, Winz M-L, Höfer K, Nübel G, Jäschke A: NAD captureSeq indicates NAD as a bacterial cap for a subset of regulatory RNAs. Nature 2014, 519:374.

34. Heil F, Hemmi H, Hochrein $\mathrm{H}$, Ampenberger F, Kirschning $\mathrm{C}$, Akira S, Lipford G, Wagner H, Bauer S: Species-specific recognition of single-stranded RNA via toll-like receptor 7 and 8. Science 2004, 303:1526-1529.

35. Vierbuchen $T$, Bang $\mathrm{C}$, Rosigkeit $\mathrm{H}$, Schmitz RA, Heine $\mathrm{H}$ : The

- human-associated archaeon methanosphaera stadtmanae is recognized through Its RNA and induces TLR8-dependent NLRP3 inflammasome activation. Front Immunol 2017, 8:1535.

Identification of TLR8 as the dominant receptor for innate immune recognition of a human-associated archeon.

36. Sander LE, Garaude J: The mitochondrial respiratory chain: a metabolic rheostat of innate immune cell-mediated antibacterial responses. Mitochondrion 2018, 41:28-36.

37. Van den Bossche J, O’Neill LA, Menon D: Macrophage immunometabolism: Where Are We (Going)? Trends Immunol 2017, 38:395-406.

38. Garaude J, Acin-Perez R, Martinez-Cano S, Enamorado M,

-• Ugolini M, Nistal-Villan E, Hervas-Stubbs S, Pelegrin P, Sander LE, Enriquez JA et al.: Mitochondrial respiratory-chain adaptations in macrophages contribute to antibacterial host defense. Nat Immunol 2016, 17:1037-1045.

Description of mitochondrial ETC adaptations in macrophages, linking innate immune recognitiono of viable bacteria and bacterial RNA to mitochondrial respiration and antibacterial defense.

39. He Y, Hara H, Nunez G: Mechanism and regulation of NLRP3 inflammasome activation. Trends Biochem Sci 2016, 41:10121021.

40. Bufe B, Schumann T, Kappl R, Bogeski I, Kummerow C, Podgorska M, Smola S, Hoth M, Zufall F: Recognition of bacterial signal peptides by mammalian formyl peptide receptors: a new mechanism for sensing pathogens. J Biol Chem 2015 290:7369-7387.

41. Kendall MM, Sperandio V: What a dinner party! mechanisms and functions of interkingdom signaling in host-pathogen associations. MBio 2016, 7 e01748.

42. Moretti J, Roy S, Bozec D, Martinez J, Chapman JR, Ueberheide B, Lamming DW, Chen ZJ, Horng T, Yeretssian G et al.: STING senses microbial viability to orchestrate stress mediated autophagy of the endoplasmic reticulum. Cell 2017, 171:809-823 e813.

43. Burdette DL, Monroe KM, Sotelo-Troha K, Iwig JS, Eckert B, Hyodo M, Hayakawa Y, Vance RE: STING is a direct innate immune sensor of cyclic di-GMP. Nature 2011, 478:515-518.

44. Wu J, Sun L, Chen X, Du F, Shi H, Chen C, Chen ZJ: Cyclic GMPAMP is an endogenous second messenger in innate immune signaling by cytosolic DNA. Science 2013, 339:826-830.

45. Bernales S, Schuck S, Walter P: ER-phagy: selective autophagy of the endoplasmic reticulum. Autophagy 2007, 3:285-287.

46. Saitoh T, Fujita N, Hayashi T, Takahara K, Satoh T, Lee H, Matsunaga K, Kageyama S, Omori H, Noda T et al.: Atg9a controls dsDNA-driven dynamic translocation of STING and the innate immune response. Proc Natl Acad Sci U S A 2009, 106:20842-20846.

47. Sauer JD, Sotelo-Troha K, von Moltke J, Monroe KM, Rae CS, Brubaker SW, Hyodo M, Hayakawa Y, Woodward JJ, Portnoy DA et al.: The N-ethyl-N-nitrosourea-induced Goldenticket mouse mutant reveals an essential function of sting in the in vivo 
interferon response to Listeria monocytogenes and cyclic dinucleotides. Infect Immun 2011, 79:688-694.

48. Rodriguez-Rodrigues N, Castillo LA, Landoni VI, Martire-Greco D, Milillo MA, Barrionuevo P, Fernandez GC: Prokaryotic RNA associated to bacterial viability induces polymorphonuclear neutrophil activation. Front Cell Infect Microbiol 2017, 7:306.

49. Papayannopoulos $\mathrm{V}$ : Neutrophil extracellular traps in immunity and disease. Nat Rev Immunol 2017, 18:134.

50. Le Bourhis L, Martin E, Péguillet I, Guihot A, Froux N, Coré M,

- Lévy E, Dusseaux M, Meyssonnier V, Premel V et al.:

Antimicrobial activity of mucosal-associated invariant T cells. Nat Immunol 2010, 11:701.

First demonstration of a selective responsiveness of MAIT cells to living bacteria.
51. Kjer-Nielsen L, Patel O, Corbett AJ, Le Nours J, Meehan B, Liu L,

-• Bhati M, Chen Z, Kostenko L, Reantragoon R et al.: MR1 presents microbial vitamin B metabolites to MAIT cells. Nature 2012 491:717-723.

Identification of microbial riboflavin metabolites as natural MAIT neoantigens, explaining their selective activation by living microbes.

52. Levy O, Suter EE, Miller RL, Wessels MR: Unique efficacy of Tolllike receptor 8 agonists in activating human neonatal antigenpresenting cells. Blood 2006, 108:1284-1290.

53. Dowling DJ, Scott EA, Scheid A, Bergelson I, Joshi S, Pietrasanta C, Brightman S, Sanchez-Schmitz G, Van Haren SD, Ninković $\mathrm{J}$ et al.: Toll-like receptor 8 agonist nanoparticles mimic immunomodulating effects of the live BCG vaccine and enhance neonatal innate and adaptive immune responses. $J$ Allergy Clin Immunol 2017, 140:1339-1350. 